\title{
Class Size Effects in Active Learning Physics Courses
}

\author{
Charles J. De Leone \\ California State University San Marcos \\ Wendell H. Potter, Catherine M. Ishikawa, Jacob A. Blickenstaff, Patrick L. Hession \\ University of California, Davis
}

With the growth of the University of California, Davis, we have been forced to seek more space for our active learning based lower-division discussion/laboratories. Our new room holds twice as many students (48) as our existing laboratories. Since we still use the smaller rooms, students taking the one-year physics sequence have a chance to experience both the larger classroom environment and the smaller one. We took advantage of this situation to study the effect of class size on students and instructors in active learning physics courses. In this paper we report on the initial results of our study of these changes and offer some insight into the differences between larger and smaller active learning settings in physics.

\section{Introduction}

The radically restructured college physics course at the University of California, Davis $^{1}$ centers around 5 hours per week of discussion/laboratory (DL), where physics students engage in active learning group work. Traditionally, a DL section enrolls 25 students and is facilitated by a single teaching assistant (TA). In the fall quarter of 2000 we were informed that campus space constraints would force us to use a larger room for the second quarter of this three quarter introductory physics sequence. This room accommodates roughly twice as many students as our original rooms. This new circumstance would force a number of modifications to our existing course, but at the same time provide a unique opportunity to study the effects of class size in an active learning based course. We report on modifications made to the course, and provide a preliminary discussion of the effect these changes had on the course, the teaching assistants, and the students.

\section{Modifications to DL}

To make use of the new room, major changes were made to our existing DL structure. In the smaller rooms, a DL section was scheduled with 25 students and one TA, with a given section meeting for two and-a-half hours twice weekly. The average TA would handle two sections, corresponding to 50 students a quarter and a net student contact time of 10 hours a week. With the switch to the larger room, the average number of students per section rose from 25 to 48. Instead of one TA per DL, we now had two, with TAs still teaching two sections, now corresponding to roughly 100 students a quarter. Students in the small room worked in groups of 5, at standard elevated lab benches or at the chalkboards on the walls. At the benches, they either sat or stood. In the new room students worked in groups of 6, at specially designed, "T" shaped tables with standard padded chairs with the new room having roughly twice as many blackboards as the smaller room.

\section{Methods of Study}

Since the switch to the larger room was only for the second quarter of the three quarter sequence, all but one of the TAs and all of the students had prior experience in the smaller rooms. Most of the students would return to the small rooms for the third quarter of the sequence. This provided a unique opportunity to make observations of the differences inherent in these two settings, and particularly on the TA and student perception of the different learning environments. 
We used a number of tools to study the change. To learn more about TA perceptions we interviewed eight TAs after they had taught in both settings. To study student perception, we used specifically designed surveys, as well as responses to standard end-of-quarter evaluations. We also used observations of the sections in both the larger and smaller rooms to complement this data.

\section{Results}

Analysis of our data yielded insight into the differences in the two learning environments. In what follows we highlight some of these differences as noticed from the TA and student perspectives.

\section{A. TA Perspective}

When switching to two TAs per section in the larger room, we decided to partner a TA who was teaching a DL for the first time with a TA who had already taught that particular DL. This partnering had the benefit of having a TA who had done that particular set of activities present in the room. For an individual TA, this meant that they would teach in their second DL section as the "experienced" TA. This linked structure guaranteed that almost all sections had an experienced TA.

This method of teaching proved to be very popular with first year TAs. Many of them expressed appreciation for the additional perspective they gained on teaching and on the details of a particular DL as a result of working with another TA. We now consider this to be a valuable aid in TA training, and plan to incorporate it in our instructor professional development program.

As for TAs who had taught for more than a year, many of them felt that the role of the subordinate TA was not well defined. A few of them said they simply ignored the subordinate TA when they acted as lead TA, and treated the class just as they would have in the 25 student DL. The issue of the role of each TA was brought up often in the TA interviews. Each pair of TAs interpreted their lead and subordinate roles differently, some splitting the room in half, others relegating the subordinate TA to simply answering small group questions, and still others sharing their lead role duties. The TAs who considered their team effort successful typically reported good communication with each other, both before and during DL.

TAs also expressed concerns relating to student numbers. Many (almost all) expressed problems with learning all of their students' names (Most TAs now have a total of nearly 100 students as opposed to 50 in the smaller rooms). Many also expressed concern over their understanding of students' current mastery level. All TAs said that getting a sense of where the class as a whole was in their understanding required talking to more students and communicating with fellow TAs.

Another source of TA dissatisfaction was the whole class discussion, where physics students have a chance to present their results to fellow students. From observations and interviews with TAs, we found that TAs in the larger room were more likely to lead wrap-up discussions themselves as opposed to having students make presentations. Quoting one TA:

"..the nature of the room made it that much harder to carry on whole class discussions. I definitely heard from fewer students..."

The reasons TAs gave for this included audibility of students in the larger room, visibility of student writing on chalk boards, student fear of speaking in front of 48 as opposed to 25 students, and the more chaotic nature of the larger classroom.

The majority of the TAs agreed that the DLs could function in the larger room, but many of the activities were more difficult simply because of the larger number of students to monitor, and the need for more organization. 


\section{B. Student Perspectives}

At the end of spring quarter 2001, students finishing both the second quarter (1st quarter small room, and $2^{\text {nd }}$ quarter large room) and third quarter $\left(1^{\text {st }}\right.$ quarter small room, $2^{\text {nd }}$ quarter large room, and $3^{\text {rd }}$ quarter small room) of the one-year sequence were asked to fill out a survey asking: "Which of the rooms suited your personal learning style better?" and "Briefly explain why you answered the way you did to the previous question." The results of this survey depended quite heavily on the room in which the students had just completed their physics course. The majority of those currently in the larger room chose the larger room over the smaller by a roughly two to one margin, with 206 choosing larger, 116 choosing smaller, and 70 stating that the room did not matter. Students currently in the smaller room gave almost exactly the opposite response, with 194 choosing the smaller room, 92 choosing the larger room, and 68 choosing neither.

An analysis of the TA approval ratings and the student comments provide some interesting insight into these answers. Those students who had TAs with an above average approval rating were more likely to choose the room in which they were just taught than those who had TAs with lower approval ratings.

Student comments on their room choice revealed a variety of reasons for choosing a particular room. Of those who chose the larger room, over a third mentioned the new chairs, the orientation of the tables, or the lighting as the reason for their choices, (e.g. "I liked not having to sit on stools..."). Students choosing the smaller rooms were less likely to mention the physical surroundings, but more likely to bring up pedagogical issues:

"(the larger room) is too big to facilitate group discussions and whole class discussions. The tables (in the big room) were better for group work, but there were too many people in each class."

We received additional insight into classsize issues from another portion of the student survey. We asked the students to estimate the number of times per DL a) the TA spoke with him or her individually, b) the TA interacted with the student's group, and c) the student spoke during whole class discussions. Our interest in these questions stems from our belief that student-TA interaction, and student verbal presentation, questioning, and discussion can facilitate development of a functional understanding of physics.

This portion of the survey was given to one cohort of students as they progressed through the 3-quarter sequence, with the first and third quarters taught in the smaller room. Responses to the first question showed that the students perceived the TAs to have had more individual interaction with them during the first quarter (small room), and that in the second quarter (large room) and the third quarter (small room), TA student interaction was roughly the same. This same pattern was noted for TA-group interaction in the DL. After comparison with some of our previous observations of new TA behavior ${ }^{2,3}$ we believe this correlates with our hypothesis that new physics TAs (who are the majority in the first quarter) spend most of their total class time interacting with individuals and groups, whereas the more experienced TAs become, the more willing they are to let individual students and groups struggle with the material before intervening.

Answers to the third question show some differences that could be attributed to the room size. Reported numbers for the question, "On average, how often do I talk during whole class discussions during a DL?" decreased noticeably in the second quarter, when the students were in the larger room. Figure 1 shows the large room $\left(2^{\text {nd }}\right.$ quarter) having a greater number of respondents averaging less than once per DL 
than those respondents in the small room $\left(1^{\text {st }}\right.$ and $3^{\text {rd }}$ quarters).

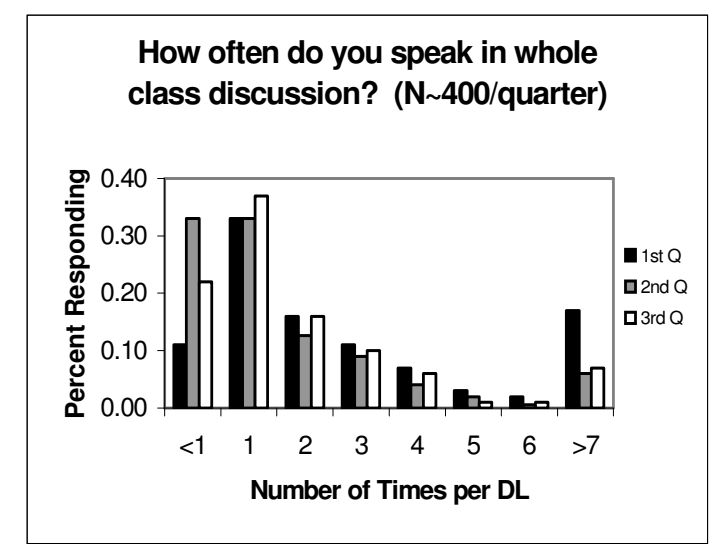

Figure 1

This result is not surprising and matches interview data from the TAs. Students and TAs both perceive that students speak less in the larger classroom. However, there is no perceived difference on the part of TAs or students concerning the number of times that students and TAs interact in the larger classroom.

\section{Conclusions}

This paper gives a brief outline of some of our findings concerning the effect of room size on student and TA perceptions of the nature of the learning environment. While we found that both students and TAs perceived benefits to either environment, we discovered that many of these benefits were not related to the number of students in the room, but rather the nature of the rooms' furnishings and lighting. Many of the new TAs viewed the partnering with a TA who had already taught a given DL as very beneficial to their teaching preparation. Both TAs and students agreed that students are less likely to speak in the larger classroom. There was no specific preference of a majority of the students for one room or another. Rather the students' choice of rooms seemed highly influenced by the learning environment that they were in at the time.
These results leave a number of questions for further study. We are very interested in collecting data on student outcomes in both environments and attempting to identify what effect, if any, this change in room size has on student learning. We also hope to more closely compare the differences in the two settings by making observations of the second quarter of the course when it is offered in a smaller room.

\section{Acknowledgments}

We would like to acknowledge the support of NSF Grant No. DUE-9354528 and the Department of Education's Fund for the Improvement of Post Secondary Education (FIPSE) Grant No. P116B70958 and the support of the physics department of the University of California, Davis.

\section{References}

${ }^{1}$ Wendell H. Potter, Charles J. De Leone, and Lawrence Coleman, "What is the Physics 7 Course?," presented at the NARST Annual Meeting, New Orleans, LA, 2000 (unpublished).

${ }^{2}$ Wendell H. Potter, Charles J. De Leone, and Catherine M. Ishikawa, "Differences in Instructor/Student Roles Detected with Behavioral Observation Tools," presented at the AAPT Summer Meeting, Guelph, Ontario, 2000 (unpublished).

${ }^{3}$ Catherine M. Ishikawa, Charles De Leone, and Wendell Potter, "Instructor Facilitation Approaches in Student-Active Labs: Two Models," presented at the AAPT Summer Meeting, Guelph, Ontario, 2000 (unpublished). 